027.7 Zeitschrift für Bibliothekskultur / Journal for Library Culture • Digital Humanities und wissenschaftliche Bibliotheken

\title{
Digital Humanities aus \\ dem Fachreferat heraus
}

Timo Glaser

Published on: Apr 20, 2021

License: Creative Commons Attribution 4.0 International License (CC-BY 4.0). 


\section{ABSTRACT}

Bibliotheken bauen seit Jahren eine forschungsunterstützende Infrastruktur und Dienstleistungen für digitale Geisteswissenschaften auf. So wichtig Retrodigitalisierung, Publikationsserver, Meta- und Forschungsdaten- sowie andere Services auch sind, so erreichen Bibliotheken jedoch einen Großteil ihrer Zielklientel aus den digitalen Geisteswissenschaften damit nicht. Ausgehend von Studien zum Information and Research Behavior in den Geisteswissenschaft wird in dem Artikel ein auf mikrologischem Blick und empathischer Praxis beruhendes Konzept entworfen, mit dem Bibliotheken aus dem Fachreferat heraus Forschende in den digitalen Geisteswissenschaften unterstützen können.

Libraries have been building research-supporting infrastructure and services for digital humanities for years. As important as digitization of print publications, institutional repositories, meta- and research data services as well as other services are, libraries do not reach a large part of their target clientele from the digital humanities. Based on studies of information and research behavior in the humanities, the article outlines a concept rooted in a micrological view and empathic practice that libraries can implement to support researchers in the digital humanities on the subject librarians' level.

\section{Status Quo}

Durch die stetige Digitalisierung ihrer Dienstleistungen verorten sich Bibliotheken zunehmend als verlässliche Dienstleisterinnen der digitalen (Geistes-)Wissenschaften in klassischen bibliothekarischen Handlungsfeldern wie der Retrodigitalisierung historischer Bestände (Thaller, 2017, 11), aber auch in jeweils neu erschlossenen Handlungsfeldern: vom Aufbau und der Bereitstellung von Publikationsservern und EJournal-Plattformen wie Open Journal Systems, über die Verwaltung von Open-AccessPublikationsfonds bis hin zu Beratungen zu Open-Science-Praktiken und Forschungsdatenmanagement samt Hosting von Forschungsdatenrepositorien. (ula, 2013).

Bibliotheken agieren damit aus einem genuinen Eigeninteresse (Retrodigitalisierung zur Sichtbarmachung und zu gleichzeitigem Schutz ihrer Bestände), reagieren auf Förderanreize Dritter (etwa der Deutschen Forschungsgemeinschaft mit ihren OpenAccess- und Forschungsdaten-Förderlinien) oder Bedarfe Einzelner 
(Wissenschaftler:innen aus den Fachbereichen, die Texte oder eine Zeitschrift auf dem Publikationsserver veröffentlichen wollen).

Damit haben Bibliotheken in den letzten Jahren ein solides Portfolio an Unterstützungsdienstleistungen, gerade auch für Forschungsprojekte aus den sich entwickelnden Digital Humanities, aufgebaut und diese oftmals in eigenen Abteilungen oder Sachgebieten institutionalisiert, in denen Dienste und Beratungen angeboten werden - von der Digitalisierung und Textauszeichnung mittels TEI, über Norm-, Metaund Forschungsdaten bis hin zu Fragen der digitalen Langzeitarchivierung. Bibliotheken sind prädestinierte Ansprechpartnerinnen, wenn es um die Etablierung einer dauerhaften Infrastruktur geht. Die zahlreichen dokumentierten Leuchtturmprojekte sind beredte Zeugen für gelungene Kooperationen zwischen Wissenschaft und Bibliothek.

Gleichzeitig lässt sich eine Parallelentwicklung ausmachen: Zahlreiche Studien zum Information und Research Behavior erwähnen, dass Bibliotheken von Wissenschaftler:innen, auch aus den Geisteswissenschaften, immer weniger als forschungsrelevant bewertet werden. (Moore \& Singley, 2019).

So sehr Bibliotheken derzeit ihre forschungsunterstützenden Aktivitäten auf den Bereich des Forschungsdatenmanagements fokussieren, so ernüchternd sind doch oftmals die Rückmeldungen:

(...): researchers do not consider the library as the first port of call when it comes to getting help with data. And they don't have to, provided they have colleagues around who are able and willing to provide them with the help they need. (Digital

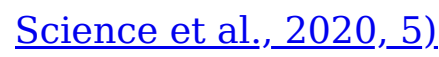

Selbst im ureigensten bibliothekarischen Feld, der Literaturversorgung und Erschließung, werden Bibliothekar:innen sogar von Geisteswissenschaftler:innen nicht als primäre Ansprechpartner:innen für Fragen der Forschung wahrgenommen:

Humanists exhibit clear patterns in their engagement with and reliance on information professionals. Crucially, humanists are more likely to rely on information professionals for support seeking primary rather than secondary information for their research. These professionals are typically located in archives, museums and other special collections not located at their home institution and they are more likely to be archivists and curators rather than librarians. Their support can be crucial to finding information, particularly when 
working with collections that have not yet been processed and/or have not been made fully available online. (...) In contrast, humanists are much less likely to consult the subject specialist librarians at their home institutions because they are

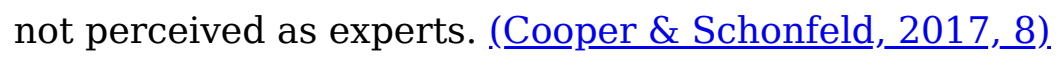

Dies widerspricht dem Zielverständnis von Bibliotheken, an möglichst vielen Punkten des (digitalen) Forschungsprozesses präsent zu sein und als Partnerin wahrgenommen zu werden. (z.B. unter vielen Vandegrift, 2018)

\section{Mit mikrologischem Blick und empathischer Praxis}

Die These des vorliegenden Artikels ist, dass dies durch die Fokussierung von Bibliotheken auf Leuchtturmprojekte mitbedingt ist: groß oder größer angelegte Forschungsvorhaben, für die Projektsteuerung, Drittmittelanträge, Personalplanung et cetera ebenso relevant sind wie eine in Förderanträgen belegte, auf Permanenz angelegte Infrastruktur als Projektergebnis.

Die traditionellen Forschungsmodi geisteswissenschaftlichen Arbeitens, das solitäre (oder in Kleingruppen vollzogene) Bearbeiten einer offenen Frage des Fachdiskurses, geraten allzu leicht aus dem Blick einer auf Infrastruktur angelegten Abteilung innerhalb der Bibliothek.

Im Folgenden möchte ich den im bibliothekarischen Diskurs bislang weniger beachteten Aspekt fokussieren, dass sich Digital Humanities nicht in Großprojekten erschöpfen, sondern dass digitale Geisteswissenschaften bereits im Kleinen betrieben werden - sowohl von Studierenden als auch von einzelnen Forscher:innen.

Des Weiteren kann mitunter die Zielklientel, die Wissenschaftler:innen aus den Digital Humanities, mit einem in einer bibliothekarischen Abteilung erarbeiteten standardisierten Serviceportfolio nicht erreicht werden, wenn dies nicht hinreichend auf divergierende Anforderungen unterschiedlichster Fächerkulturen hin ausgerichtet ist.

Es ist nicht leicht, eine Service-Infrastruktur zur Unterstützung digitaler geisteswissenschaftlicher Forschung aufzubauen, wenn das Gegenüber eine schwer fassbare Chimäre bleibt. Schließlich ist „Digital Humanities“ kein wohldefinierter Begriff, sondern ein „Umbrella Term“, der je nach Kontext verschiedene Bedeutungen annimmt: Sei es, dass man Digital Humanities als eigenes Fach mit eigener Hermeneutik und Methodik versteht, das dann eher geisteswissenschaftlich, informationswissenschaftlich oder computerwissenschaftlich verstanden werden kann, 
sei es, dass Digital Humanities jeweils als Teildisziplin etablierter Wissenschaften verstanden wird, etwa Computerlinguistik oder Archäoinformatik. Und selbst innerhalb einer Disziplin kann die „digitale Tradition“ abhängig von der wissenschaftlichen Herkunft der Forschenden äußerst heterogen sein: Das Fundament allein der digitalen Literaturwissenschaften zum Beispiel kann in den Sozialwissenschaften, den Kognitionswissenschaften oder der Computerlinguistik gelegt sein. (ㅍp라, 2018, $\underline{5}$ ).

Die vorangegangenen aus der Literatur abgeleiteten Erwägungen führen dazu, Digital Humanities nicht nur als bibliothekarische Infrastrukturherausforderung zu sehen, sondern als originär fachwissenschaftliche Herausforderung. Sich den Digital Humanities mit „mikrologischem Blick und empathischer Praxis“ $\underline{1}$ zu nähern, (re)loziert sie in die fachliche Supportstruktur, die Bibliotheken über Jahrzehnte mit dem Fachreferat aufgebaut haben.

Der „mikrologische Blick“ richtet sich dabei explizit auf die alltägliche Praxis der Digitalisierung geisteswissenschaftlichen Arbeitens allgemein, wie sie von einzelnen Wissenschaftler:innen und Studierenden gelebt oder angestrebt wird, die „empathische Praxis“ verweist darauf, dass das eigene digitale Forschen wesentliche Voraussetzung ist, die fachbezogene Betreuung nachhaltig zu verbessern und die avisierte Omnipräsenz der Bibliothek im Forschungszyklus zu erreichen. (vgl. auch (King, 2018, 45) )

\section{Digital Humanities als Vermittlungsaufgabe}

Digital Humanities sind nicht nur Großprojekte, die Digital-Humanities-Praxis fängt bereits im Kleinen an, sei es bei den einzelnen Wissenschaftler:innen, sei es bereits im Studium: Methoden oder Techniken computergestützten Arbeitens können (oder könnten) auch bereits von Studierenden eingesetzt werden, um ihre Fragestellungen zu beantworten. $\underline{2}$

Es finden sich jedoch an Universitäten kaum Orte, an denen Studierende der Geisteswissenschaften diese Techniken lernen können. In einzelnen Studiengängen gibt es freilich fachspezifische Angebote, in reguläre Module oder Lehrveranstaltungen eingebettete Workshops oder Ähnliches werden allerdings eher selten von fachfremden Studierenden wahrgenommen. Fachübergreifende Workshops gibt es gelegentlich in Graduiertenschulen, diese stehen dann allerdings den meisten Studierenden nicht offen. 
Wollen Studierende Programmiersprachen lernen, um mit deren Hilfe Textdokumente automatisiert zu analysieren, oder Techniken kennenlernen, wie sie aus Digitalisaten computerlesbare Textdokumente erstellen können, wie sie Texte so aufbereiten, dass aus ihnen berechenbare Datensammlungen werden, sind sie auf sich allein gestellt. Freilich, freie Angebote gibt es zuhauf online (etwa über edX, Programming Historian, forText, DARIAH et cetera) oder auch in Form von Lehrbüchern, aber zum einen hilft es Studierenden in geleiteten Veranstaltungen, sich grundständig neue Arbeitstechniken anzueignen, zum anderen ist nicht immer einsichtig, was bereits mit wenigen digitalen Grundkenntnissen alles möglich ist. Workshops können hier auch die Funktion übernehmen, die Teilnehmenden auf neue Ideen zu bringen, wie sie mit digitalen Techniken in ihrem Fach, mit ihren Quellen Fragen neu stellen und beantworten können, und so ihren Methodenkoffer erweitern. (uㅣlen, 2018, Kapitel 1).

Digital Humanities erweisen sich hier zuvorderst als Informationskompetenz-Problem, weniger als Infrastrukturproblem. Diese Überlegungen führten dazu, an der Universitätsbibliothek Marburg ein Digital Humanities Learning Lab einzurichten, das solche basalen Digital-Humanities-Kompetenzen speziell an

Geisteswissenschaftler:innen vermittelt. $\underline{3}$

Das Herangehen an Digital Humanities als eine Informationskompetenz-Aufgabe hat konkret mehrere Nebeneffekte gezeitigt:

Zum einen erhöht es die Sichtbarkeit der Bibliothek als Ansprechpartnerin für DigitalHumanities-Themen und -Fragen und ermöglicht so Vernetzungen über enge Fächergrenzen hinaus, jenseits von großen Forschungsprojekten, die eine Infrastruktur-Unterstützung benötigen.

Teilnehmer:innen der Workshops lernen die Bibliothek als Ansprechpartnerin kennen und kommen bei zukünftigen Forschungsfragen auf die Bibliothek zu, wenn sie etwas mit digitalen Methoden zu tun haben, auch über die konkreten Inhalte der Workshops hinaus.

Durch die Ankündigung der Workshops auf den Webseiten der Bibliothek und in SocialMedia-Posts wird die Bibliothek auch durch Websuchen nach Digital-HumanitiesThemen sichtbar und es ergeben sich Beratungssituationen, Forschungskooperationen und Teilnahme an Lehrveranstaltungen, die nicht antizipierbar sind, und helfen der Bibliothek, Kontakte zu ansonsten nicht erreichten, bibliotheksfernen Wissenschaftler:innen (punktuell) aufzubauen. 
Nicht zuletzt erweitert das Wissen um Techniken der digitalen Geisteswissenschaften die bibliothekarische Handlungsfähigkeit und Methodenkompetenz:

- Die Einarbeitung in OCR-Tools ermöglicht es zum Beispiel alte, noch mit Schreibmaschine geschriebene Haussystematiken zu aktualisieren und effizienter zu nutzen;

- mit „Regulären Ausdrücken“ wird es möglich, schneller semistrukturierte Informationen aus (Text-)Dokumenten zu extrahieren;

- die Nutzung der statistischen Programmiersprache R anstelle von Excel kann jährlich wiederkehrende Analysen der Ausleihdaten vereinfachen;

- mit Python- oder R-Skripten können ganze Verzeichnisse mit Textdokumenten eingelesen und zum Beispiel mit Topic-Modeling ausgewertet und annotiert werden, um schneller Themen in einzelnen Dokumenten (zum Beispiel in Sitzungsprotokollen) zu identifizieren.

Digital Humanities als Aufgabe der Vermittlung von Informationskompetenz zu betrachten und die Einarbeitung in entsprechende Techniken anzustoßen, verhilft zu einem fundierten Überblick in der Breite über Techniken der Digital Humanities und erweitert die eigene Beratungs- und Methodenkompetenz innerhalb der Bibliothek.

\section{Digital Humanities als Forschungsaufgabe}

Ein tieferer Einblick in einzelne Methoden und Techniken der Digital Humanities wird darüber hinaus ermöglicht, wenn diese nicht nur in Workshops gelehrt, sondern in eigener Forschung angewandt werden. Wenn sich Bibliothekar:innen durch eigenständige Forschung den Bereich der Digital Humanities weiter erschließen, kann dies in mehrfacher Hinsicht Vorteile mit sich bringen.

Zunächst gewinnt man durch die praxisnahe, intensive Beschäftigung mit einigen wenigen digitalen Methoden und Werkzeugen einen vertieften Einblick, was mit diesen alles möglich ist und vor allem was nicht.

Für ein kleines Forschungsprojekt zum antiken ethnographischen Diskurs wurden antike griechische Texte zusammengestellt und mithilfe von Python- und R-Skripten und der Software Mallet analysiert, um gemeinsame Topics auszumachen, die genutzt werden, um religiöse Sondergruppen zu charakterisieren. Erst die intensive Beschäftigung macht auf die zahlreichen Fallstricke aufmerksam: von der Zugänglichkeit und Nachnutzbarkeit der Quellenschriften, über unterschiedliche TEIAuszeichnungen und Unicode-Codierungen griechischer Buchstaben und Akzente bis hin zu Detailfragen der Dependencies von Python Libraries und der Programmierung 
von Skripten zur Text- und Datenauswertung und Visualisierung mit den tidyverse RPackages.

Im geschützten Raum eines Workshops mit zwar praxisnahen aber dennoch vereinfachten Beispielen zur Vermittlung der Methodenkenntnisse stößt man selten auf die konkreten Herausforderungen des digitalen Forschungsalltags. Diese selbst erlebt zu haben und Lösungen dafür gefunden zu haben, hilft in der Beratung ebenso wie in zukünftigen Schulungskontexten.

Nicht zu vernachlässigen ist darüber hinaus der wiedergewonnene Einblick in den konkreten Forschungsalltag. Wie schnell geraten die Arbeitsprozesse der Wissenschaft aus dem bibliothekarischen Blickfeld. Dienstleistungen von Bibliotheken, die an den tatsächlichen Erfordernissen der Forschung vorbeigehen, sind nicht selten die Konsequenz. Nicht zuletzt mit Studien zum Information und Research Behavior, die aus dem angloamerikanischen Raum kommend auch im deutschsprachigen Bibliotheksdiskurs vermehrt aufgegriffen werden, versucht die Bibliothekswissenschaft, einen neuen Blick dafür zu gewinnen.

Eigenständig wieder forschend tätig zu werden, und sei es auch nur punktuell mit kleinen Projekten, lässt den Forschungsalltag erleben und erhöht die Kommunikationsfähigkeit mit den Wissenschaftler:innen. Wenn die Forschung im „eigenen“ Fach erfolgt, kann dies darüber hinaus zu einer tieferen Kooperation und Kommunikation mit dem eigenen Fachbereich führen, zum Teil sogar zu Teilnahmen an Forschungskolloquien, in denen die Angehörigen des Fachbereiches aktuelle Forschungsprojekte vorstellen und diskutieren. Diese Teilnahmen erhöhen wiederum die Sichtbarkeit der Bibliothek in den Fachbereichen, bringen Bibliothekar:innen ins Blickfeld der Forschenden und ermöglichen weitere Kooperationen.

Fachwissenschaftler:innen benötigen Bibliothekar:innen (auch aus dem eigenen Fach) freilich nicht für den Fachdiskurs, dazu greifen sie lieber auf ihr eigenes Netzwerk zurück:

In contrast, humanists are much less likely to consult the subject specialist librarians at their home institutions because they are not perceived as experts. Even if, as appears to be increasingly the case, subject specialists have advanced degrees in the relevant subject area, subject expertise at a disciplinary level is not what is being sought. Rather, for research support humanists are looking for engagement at the level of their own subdiscipline, which is rarely available through the library. (․ㅡㄹㅡ \& Schonfeld, 2017, 
Gleichwohl kann ihre Forschung durch das spezifische bibliothekarische Profil gewinnen, wenn dieses in die jeweilige Fachkultur übersetzt werden kann. Diese Funktion der Fachreferent:innen als Brückenbauer:innen zwischen Bibliothek und Infrastrukturfragen auf der einen, und der Fachwissenschaft auf der anderen Seite kann allerdings nur wahrgenommen werden, wenn Fachreferent:innen auch mit ihrer wissenschaftlichen Fachkompetenz und überhaupt als Kooperationspartner:innen wahrgenommen werden. $\underline{4}$

Neben der Forschung im eigenen Fach kann Forschung auch mit Bibliotheksbeständen erfolgen. In der bereits oben zitierten Studie von Cooper und Schonfeld wurde von Geisteswissenschaftler:innen betont, dass sie weniger Bibliothekar:innen, als vielmehr Archivar:innen und Kurator:innen benötigen, um Zugang zu Spezialbeständen zu bekommen, die zum Teil noch nicht oder nur marginal erschlossen sind. (․oper \& Schonfeld, 2017,, 8).

In Forschungsbibliotheken gehört die forschende Arbeit mit ihren Beständen und die Kooperation mit externen Forscher:innen auch zum Tagesgeschäft der Bibliothekar:innen. (Gleixner \& Steyer,_2021). Aber auch Universitätsbibliotheken sollten diese Erforschung der eigenen Bestände verstärkt in den Blick rücken und „ihre Sammlungen und Objekte wieder lesen lernen“ Schröter,_2017. Durch solche aktive Forschungsarbeit, die im fachwissenschaftlichen Diskurs gegründet ist, können Bibliothekar:innen nicht zuletzt lernen zu beurteilen, wie Praktiken und Standards der Retrodigitalisierung und Metadatenerschließung Forschungsfragen beeinflussen, ermöglichen oder verhindern. (vgl. Schröter, 2017 und auch Gleixner \& Steyer, 2021 zum Konzept der „erschließenden Forschung“) Es ist noch nicht allzu lange her, dass Bibliotheken sich in der Retrodigitalisierung damit begnügten, Bilder ihrer historischen Bestände ohne offene Lizenz-Angaben zur Verfügung zu stellen und damit für die Forschung unbrauchbare und kaum genutzte Digitalisate zu produzieren.

Die Erforschung der eigenen Bestände ermöglicht des Weiteren, besondere Sammlungen zu identifizieren, zu bewerten, digital zugänglich zu machen und zu präsentieren. (Dang, 2018, 149) . Nicht zuletzt durch solch profilierte Bibliotheksbestände und forschende Bibliotheksarbeit rücken Bibliothekar:innen - aus Sicht der Wissenschaftler:innen - dann wieder näher an die gesuchten Kurator:innen und Archivar:innen heran und werden als Kooperationspartner:innen sichtbar. 


\section{Vom Servicemodell zu forschenden Bibliothekar:innen}

Im US-amerikanischen Bibliotheksbereich lässt sich eine Tendenz ausmachen, nach der forschende Bibliothekar:innen vermehrt gesucht und benötigt werden. Das traditionelle Servicemodell zur Unterstützung von Wissenschaftler:innen erscheint dort nicht zukunftsweisend und wird durch Bibliothekar:innen ergänzt, die auch als Forschende kompetent sind und so mit den Wissenschaftler:innen kollaborieren können:

A move away from a service model where librarians are only available to meet the needs and give support to researchers has occurred. In this context where digital humanities is being carried out in libraries, librarians are sought who are collaborators in the research, giving rise to a new (or more frequently seen) library position, that of the digital scholarship librarian. (King, 2018, 45).

Inwieweit diese Tendenz auch im deutschsprachigen Bibliotheksbereich um sich greifen wird, bleibt abzuwarten. Ausgehend von der Beobachtung, dass Bibliotheken aus Sicht der Forschenden im Forschungsprozess nicht so zentral sind, wie sie selbst es intendieren, war die Absicht des Artikels, für diese Profilierung des Fachreferates eine Lanze zu brechen.

Gilt dies über verschiedene Fachkulturen hinaus, so erweist sich gerade auch für eine Forschungsunterstützung im Bereich der Digital Humanities die Frage, „Welche Services werden von wem benötigt?", als nicht trivial. Zu heterogen ist die digitale Verwurzelung und Arbeitsweise in den einzelnen geisteswissenschaftlichen Fächern. Allzu oft begnügen sich Bibliotheken dann damit und verbuchen es als „Erfolg“, wenn sie in einigen Drittmittelprojekten als Partnerin involviert sind und eine Infrastruktur (möglichst noch als „Leuchtturm“ ausgewiesen) mit aufbauen. Zu viele potentielle Forschungspartner:innen werden so nicht erreicht.

Demgegenüber wurde der Ansatz skizziert, an die Digital Humanities mit mikrologischem Blick und empathischer Praxis heranzugehen: Digital Humanities nicht nur als groß angelegte Editionsprojekte zu verstehen, sondern als Arbeitsalltag im ganzen Spektrum von geisteswissenschaftlicher Forschung, Lehre und Studium.

Digital Humanities als Aufgabe der Informationskompetenz zu sehen, hilft Bibliothekar:innen auch dabei, einen Überblick über die zahlreichen digitalen Ansätze, Methoden und Werkzeuge zu gewinnen. 
Eigenständig wieder in die Forschung einzutreten, kann dieses oberflächliche Wissen punktuell vertiefen und ermöglicht damit zugleich, das gesamte Feld der Digital Humanities besser zu verstehen und einen aktuellen Blick in den forschenden Arbeitsalltag zu bekommen. Letzteres kann dazu führen, den Kontakt in die eigenen Fächer auszubauen, neue Kontakte zu knüpfen, die Bibliothek mit ihren Beständen und ihren Mitarbeitenden auch für die Forschung wieder sichtbar zu machen.

\section{Footnotes}

1. Mit dieser Wendung beschreibt Kristian Fechtner den Ansatz einer „Praktischen Theologie des Subjektes“ des Marburger Theologen Henning Luther, vgl. Fechtner, K. (1992). Mikrologischer Blick und Empathische Praxis: Hinweise zu Henning Luthers Praktischer Theologie des Subjekts. Praktische Theologie, 27(3), 184-193. https://doi.org/10.14315/prth-1992-0305.

2. "Even if grad students are not interested in DH as a discipline, per se, there is a strong likelihood that their academic futures will require some digital competence." (Battershill, C., \& Ross, Shawna (2017). Using Digital Humanities in the Classroom: A Practical Introduction for Teachers, Lecturers, and Students. London, New York: Bloomsbury Academic, 148)

3. Vgl. Glaser, T. (2018). Wie können Bibliotheken Studierende fit für die Digital Humanities machen? ABI Technik, 38(3), 282. https://doi.org/10.1515/abitech-20183011. Zum Standardprogramm gehören Workshops zur Textanalyse mit Python und R, OCR-Techniken, Datenextraktion mit Regulären Ausdrücken und Webscraping, Open Refine und Git(hub/lab) etc. sowie Grundlagen des

Forschungsdatenmanagements. $\subseteq$

4. Vgl. Hartsell-Gundy, A., Braunstein, L., Golomb, L., \& Langan, K. (2015). Digital humanities in the library: challenges and opportunities for subject specialists. Chicago: Association of College and Research Libraries,12-14, http://www.ala.org/acrl/sites/ala.org.acrl/files/content/publications/booksanddigitalres $=$ 
ources/digital/9780838987681_humanities_OA.pdf zur Rolle der Subject Librarians als Brückenbauer auf verschiedenen Ebenen. $\subseteq$

\section{Citations}

1. Thaller, M. (2017). Geschichte der Digital Humanities. In F. Jannidis, H. Kohle, \& M. Rehbein (Eds.), Digital Humanities (pp. 3-12). Stuttgart: J.B. Metzler. https://doi.org/10.1007/978-3-476-05446-3_1

2. Sula, C. A. (2013). Digital Humanities and Libraries: A Conceptual Model. Journal of Library Administration, 53(1), 10-26.

https://doi.org/10.1080/01930826.2013.756680 3. Moore, M., \& Singley, E. (2019). Understanding the information behaviors of doctoral students: An exploratory study. Portal: Libraries and the Academy, 19(2), 279-293. https://doi.org/10.1353/pla.2019.0016

4. Digital Science, Hahnel, M., McIntosh, L. D., Hyndman, A., Baynes, G., Crosas, M., Nosek, B., Shearer, K., van Selm, M., Goodey, G., \& Nature Research. (2020). The State of Open Data 2020. https://doi.org/10.6084/m9.figshare.13227875.v2 5. Cooper, D., \& Schonfeld, R. C. (2017). Rethinking Liaison Programs for the Humanities. Ithaka $S+R$. https://doi.org/10.18665/sr.304124 6. Vandegrift, M. (2018). Designing Digital Scholarship Ecologies. LIS Scholarship Archive. https://doi.org/10.31229/osf.io/93zvb $ヒ$

7. Piper, A. (2018). Enumerations: data and literary study. Chicago, London: University of Chicago Press. $\_$ 8. King, M. (2018). Digital Scholarship Librarian: What Skills and Competences are Needed to be a Collaborative Librarian. International Information \& Library Review, 50(1), 40-46. https://doi.org/10.1080/10572317.2017.1422898 ヒ 9. Mullen, L. A. (2018). Computational Historical Thinking. With Applications in R. Retrieved from https://dh-r.lincolnmullen.com/introduction.html $\boxminus$ 10. Gleixner, U., \& Steyer, T. (2021). Forschung in Bibliotheken. ZfBB, 68(1), 27-37. https://doi.org/10.3196/186429502068149

11. Schröter, M. (2017). Teaching Libraries zwischen Vermittlung fachwissenschaftlicher Informationskompetenz und der Unterstützung einer 
Propädeutik für die Digital Humanities. O-Bib. Das Offene Bibliotheksjournal, 4(1), 76-94. https://doi.org/10.5282/o-bib/2017H1S76-94ㅌ

12. Gleixner, U., \& Steyer, T. (2021). Forschung in Bibliotheken. ZfBB, 68(1), 27-37. https://doi.org/10.3196/186429502068149

13. Dang, S.-M. (2018). Digital Tools \&Big Data: Zu gegenwärtigen

Herausforderungen für die Film-und Medienwissenschaft am Beispiel der feministischen Filmgeschichtsschreibung. MEDIENwissenschaft: Rezensionen/ Reviews, (2-3), 142-156. https://doi.org/10.17192/ep2018.2-3.7836 